\title{
Competing risks: Competing questions
}

\author{
Jeevanantham Rajeswaran, $\mathrm{PhD},{ }^{\mathrm{a}}$ and Eugene $\mathrm{H}$. Blackstone, $\mathrm{MD}^{\mathrm{a}} \mathrm{b}$
}

\footnotetext{
From the Departments of ${ }^{\mathrm{a}}$ Quantitative Health Sciences and ${ }^{\mathrm{b}}$ Thoracic and Cardiovascular Surgery, Cleveland Clinic, Cleveland, Ohio.

Disclosures: Authors have nothing to disclose with regard to commercial support.

Received for publication March 6, 2017; accepted for publication March 6, 2017; available ahead of print April 7, 2017.

Address for reprints: Jeevanantham Rajeswaran, $\mathrm{PhD}$, Cleveland Clinic Department of Quantitative Health Sciences, 9500 Euclid Ave, JJN3, Cleveland, OH 44195 (E-mail: rajeswj@ccf.org).

J Thorac Cardiovasc Surg 2017;153:1432-3

$0022-5223 / \$ 36.00$

Copyright (c) 2017 by The American Association for Thoracic Surgery

http://dx.doi.org/10.1016/j.jtcvs.2017.03.017
}

Eighteenth-century England: Progress in preventing smallpox by inoculation is encouraging, but comes with a price- $10 \%$ mortality. As the search for low-risk protection intensifies, what will be the economic impact of eradicating the disease? Daniel Bernoulli tackles this question by introducing the concept of competing causes of death, ${ }^{1}$ calculating mortality if death from smallpox is removed. Just 36 years later, Edward Jenner inoculates 8-year-old James Phipps with pus from cowpox blisters on milkmaid Sarah Nelmes.

As noted by Huebner and colleagues, ${ }^{2}$ during follow-up after cardiac surgery, patients can experience several clinical events besides death. The probability of their occurrence is usually estimated by the Kaplan-Meier method. This method provides a "pure" result, meaning that it produces probability estimates as if no competing events confounded its occurrence. For example, to the question "What is the probability of stroke after aortic valve replacement?" the Kaplan-Meier method produces probabilities across time based on the instantaneous risk of stroke. However, in older patients, even if the risk of stroke remains the same, fewer strokes are counted because more patients die first. The question now might be, "What is the likelihood of having a stroke before one dies?" When the probability of occurrence of 1 event, such as death, hinders or alters the occurrence of other events, such as stroke, this is called "competing events" or "competing risks." 3 Unlike the "pure" Kaplan-Meier method, competing-risk estimates can be thought of as being confounded by other competing risks. Indeed, answers depend on what and how many competing risks you choose to consider. Thus, if one is interested in the likelihood of reaching a Fontan operation after birth with a hypoplastic left heart, one may need to consider death after first- and second-stage palliation, transplantation, and occasional 2- and 1.5-ventricle repairs. Typically, the likely occurrence of all competing events and freedom from any such occurrence are graphically portrayed by the cumulative incidence function. At every point in time, the likelihood of all these sums to $100 \%$.

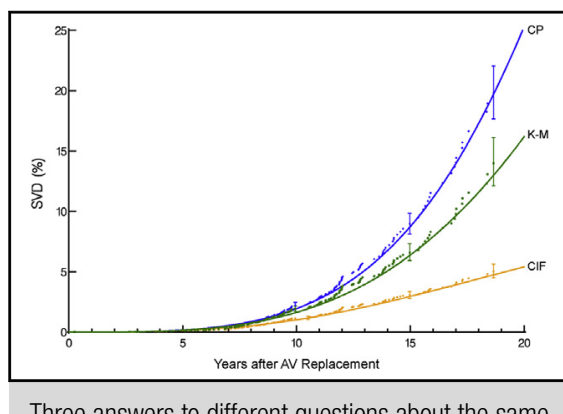

Three answers to different questions about the same SVD data.

Central Message

Competing events are observed frequently after cardiac surgery, and there seems to be competing statistical methods for their analysis. However, the choice of method depends on the research question.

See Article page 1427.

However, some confusion has arisen when probability estimates from a Kaplan-Meier analysis have been compared with competing-risk cumulative-incidence estimates with implications that one is right and the other is wrong. It's not a question of right or wrong, or hypothetical versus confounded; rather, the method chosen for analysis must match the research question. ${ }^{4}$

Consider 3 research questions:

1. What is the intrinsic durability of a heart valve bioprosthesis assessed by explant for structural valve deterioration (SVD)?

2. Given the competing risks of death and explant for other causes, how likely is it that patients will live long enough to require explant of a deteriorated bioprosthesis?

3. In living patients who have not experienced explant for other causes by a given time point, what is the probability at that time of explant for SVD?

Although Huebner and colleagues ${ }^{2}$ acknowledge the cumulative incidence function will not answer all questions, it is important to understand the appropriate analysis for each.

The answer to the first question requires that we consider who the "actor" is. We argue it is the prosthesis. ${ }^{5}$ Therefore, we recommend a "pure" analysis of durability with the confounding effects of death and explant for other causes ignored: a Kaplan-Meier analysis (Figure 1, $K-M$ ).

The answer to the second question requires that we simultaneously account for the competing possibility that 


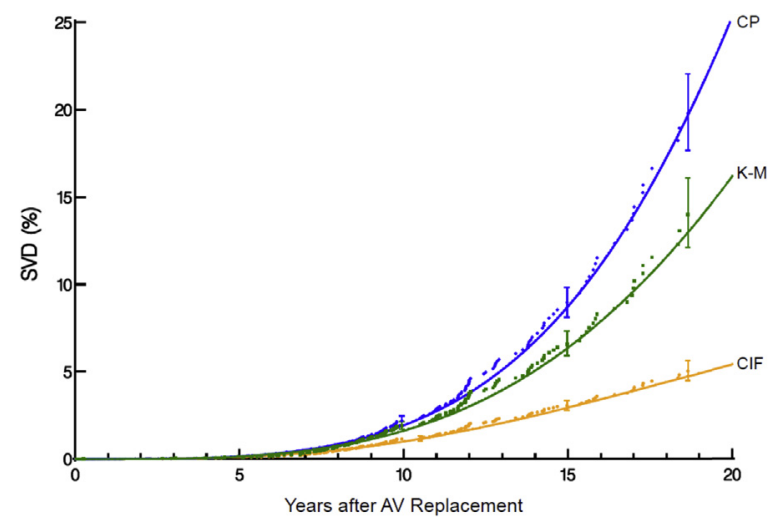

FIGURE 1. Explant for SVD of a bioprosthetic aortic valve. The KaplanMeier curve depicts the probability of this event not confounded by death or explant for other causes, thus demonstrating the durability of the valve. The cumulative incidence function curve depicts the likelihood of patients requiring explant for SVD given the competing risk of death or explant for other causes. The conditional probability curve is the conditional cumulative incidence function based on cumulative incidence functions, depicting the likelihood of explant for SVD given that no competing risks have occurred yet. $S V D$, Structural valve deterioration; $C P$, conditional probability; $K-M$, Kaplan-Meier; $C I F$, cumulative incidence function; $A V$, aortic valve.

patients may die or undergo explantation for other causes before the prosthesis is explanted for SVD: a typical competing-risk analysis displaying cumulative incidence functions (Figure 1, CIF).

The answer to the third question is not addressed by Huebner and colleagues. ${ }^{2}$ It is the conditional probability of explant for SVD given that the competing events have not yet occurred, and the quantity graphed would be the conditional incidence function defined by Pepe and Mori ${ }^{6}$ (Figure 1, CP).

Risk factor modeling also differs according to the question. For the "pure" effect model, analysis is of "cause-specific hazard," in which variable selection and model fitting can be performed using semiparametric or parametric models. For the confounded effect model, analysis can be performed on "hazard of subdistribution," as described by Fine and Gray. ${ }^{7}$ Huebner and colleagues, ${ }^{2}$ detailed discussion and illustration of such a model strengthens their article. Although Huebner and colleagues ${ }^{2}$ have performed some focused risk-factor analyses for all competing risks considered simultaneously, variable selection methods for this model are not discussed. Only a few such general methods exist, such as methodology based on random survival forests proposed by Ishwaran and colleagues. ${ }^{8}$ Of note, such multivariable analysis methods, at least to some extent, account for the possibility of informative censoring.

Confusion about when to use a noncompeting risk method, a competing risk method, or a conditional competing risk method is alleviated by carefully considering the research question and applying the appropriate method of analysis and display.

\section{References}

1. Bernoulli D. Essai d'une nouvelle analyse de la mortalité causée par la petite verole et des avantages de l'inoculation pour la prévenir. In: Histoire avec les Memoire. Paris: Acad Sci; 1760:1-45.

2. Huebner M, Wolkewitz M, Enriquez-Sarano M, Schumacher M. Competing risks need to be considered in survival analysis models for cardiovascular outcomes. $J$ Thorac Cardiovasc Surg. 2017;153:1427-31.

3. Pintilie M. Competing Risks: A Practical Perspective. West Sussex, England: John Wiley \& Sons Ltd; 2006.

4. Bodnar E, Blackstone EH. Editorial: an 'actual' problem: another issue of apples and oranges. J Heart Valve Dis. 2005;14:706-8.

5. Johnston DR, Soltesz EG, Vakil N, Rajeswaran J, Roselli EE, Sabik JF III, et al. Long-term durability of bioprosthetic aortic valves: implications from 12,569 implants. Ann Thorac Surg. 2015;99:1239-47.

6. Pepe MS, Mori M. Kaplan-Meier, marginal or conditional probability curves in summarizing competing risks failure time data? Stat Med. 1993;12: 737-51.

7. Fine JP, Gray RJ. A proportional hazard model for the subdistribution of a competing risk. J Am Stat Assoc. 1999;496-509.

8. Ishwaran H, Gerds TA, Kogalur UB, Moore RD, Gange SJ, Lau BM. Random survival forests for competing risks. Biostatistics. 2014;15:757-73. 